\title{
El Tiempo, uso y abuso
}

\section{Delma R. Álvarez ${ }^{1}$ y Giovanni P. Lobo ${ }^{2}$}

(1) Facultad de Arquitectura - Universidad del Atlántico. Km 7 Antigua vía Puerto Colombia, Colombia

(correo-e: delmarocha@mail.uniatlantico.edu.co)

(2) Facultad de Ciencias de la Educación, Universidad del Atlántico, Km 7 Antigua vía Puerto Colombia, Colombia (correo-e: gpolifroni@mail.uniatlantico.edu.co)

Recibido Ago. 14, 2019; Aceptado Oct. 14, 2019; Versión final Ene. 4, 2020, Publicado Abr. 2020

\section{Resumen}

Este artículo analiza y discute acerca de la razón fundamental por la que los estudiantes universitarios de la facultad de arquitectura de una universidad privada de Colombia, no utilizan bien el tiempo, en todas sus dimensiones. Se estudian las causas o distractores que impiden su racionalización o manejo inteligente, siendo este recurso importante y básico para los profesionales exitosos de este nuevo milenio. El artículo es de tipo descriptivo-reflexivo, cuyo objetivo es el demostrar la importancia del uso del tiempo. Basado en los antecedentes teóricos y resultados empíricos obtenidos en la investigación, se concluye que se debe planificar en forma adecuada la distribución y uso del tiempo y ser consciente que siempre se puede mejorar el uso del tiempo.

Palabras clave: uso del tiempo; tiempo biológico; tiempo psicológico; tiempo real; planificación; preempción

\section{Time, use and abuse}

\section{Abstract}

This article analyzes and discusses about the fundamental reason on why university students of the faculty of architecture, of a private university in Colombia, do not make appropriate use of time. The study shows the causes or distractions that prevent their rationalization or intelligent management, being this an important and basic resource for the successful professionals of this new millennium. The article is of a descriptivereflective type, whose objective is to demonstrate the importance of the use of time. Based on the theoretical background and empirical results obtained in the investigation, it is concluded that distribution and use of time must be appropriately planned and students must me conscious that use of time can always be optimized. 


\section{INTRODUCCION}

El tiempo, es un concepto que fue abordado por los antiguos iranios. En esta población, se tenía la creencia, de que existían dos clases de tiempo: Un tiempo indefinido o infinito que los griegos tradujeron por cronoj, sin principio ni fin; y un tiempo cósmico inspirado por la mitología griega conocido como Kronos, cuya particularidad era la de devorar a sus hijos (Zubiri, 1976). Esta idea del tiempo, fue denominada por Platón, como Eon, traducido al concepto eternidad, donde se engendraban las cosas para luego ser tragadas. También Eon, por su parte, era considerado, como el tiempo propio de cada cosa, el tiempo del ser vivo, su edad y la duración de la vida (Zubiri, 1976).

En el ámbito de la filosofía, existen posiciones encontradas acerca del tiempo, y comúnmente, su conceptualización, se relaciona con el espacio, los eventos, un antes y después entre otros aspectos. Todo ello, acudiendo al grado de relatividad en la concepción del tiempo. Santo Tomás de Aquino, tuvo una noción particular del tiempo en su identificación del movimiento que ocurre en un antes y un después, Aquino, hizo la siguiente afirmación en la física:... distinguimos "antes" y "después" principalmente en su lugar; y ahí estamos Distinguirlos por su posición relativa. Pero el movimiento también debe tienen en él una distinción de "antes" y "después" análogamente a eso en magnitud. El orden de "antes" y "después" que está en proceso es, existencialmente, el proceso. Aunque, en efecto, cuál es la distinción. Entre "antes" y "después" es diferente de qué es un proceso. (Blackwell, et al., 1963).

También Platón, identifico al tiempo como un proceso, y específicamente, con la revolución celestial. Aristóteles se opuso a este punto de vista en varios aspectos. Primero, un cambio o proceso tiene una ubicación en el espacio, y el tiempo no. Segundo, el movimiento es rápido o lento, pero hay o no hay un sentido literal, en el que el tiempo sea rápido o lento. De hecho, se define "rápido" y "lento" en términos de tiempo: "Eso es" rápido "en el que hay mucho en poco tiempo " (Hope, 1961, p.10). Sin embargo, el tiempo no es conceptualmente independiente del cambio. El argumento que Aristóteles usa para establecer esto es fenomenológico: no se puede percibir el tiempo como tal; se debe estar conscientes del paso del tiempo solo a través del cambio o movimiento discernidor. También se argumenta, que el tiempo no es un proceso puro, sino que es un aspecto numerable de eso. Esto se indica, por el hecho de que, al discriminar "más" o "menos" por número, por lo que se discriminas el movimiento "más" o "menos" por tiempo (Frassen, 2013).

El maestro Isaac Barrow, consideró el problema que había llevado a Aquino a la distinción entre imaginario y tiempo real, pero la reacción de Barrow fue más radical. Su solución fue rechazar por completo la idea aristotélica de que el tiempo es un aspecto del movimiento (Cajori, 1960, p. 6). Barrow pregunta explícitamente, si había tiempo antes de la creación (es decir, si el instante de creación es o no es el primer instante) (Child, 1916). Para Barrow, la calidad del tiempo no depende esencialmente de ninguno. Si las cosas corren o se detienen, ya sea que se duerma o se despierte, el tiempo fluye en su tenor uniforme. Este autor considera, que todas las estrellas han permanecido fijas desde su nacimiento, por tanto, nada hubiera sido perdido al tiempo, y tanto tiempo habría durado esa quietud como continuó el flujo de este movimiento. Según Barrow, el espacio y el tiempo existen independientemente de los cuerpos materiales 0 acontecimientos físicos, pero no independientemente de Dios (Burrt, 1932).

Isaac Newton, aceptó la teoría del tiempo de Barrow. En el famoso Scholium a su Philosophiae Naturalis Principia. Mathematica Newton afirma: Porque los tiempos y los espacios son, por así decirlo, los lugares también de sí mismos y de todas las demás cosas. Todas las cosas son colocadas en el tiempo en orden de sucesión; y en el espacio como por orden de situación (Cajori, 1960). Locke hace mención, de que el mundo fue creado por un cierto número de revoluciones del sol antes de la creación del sol. Sin embargo, se considerar que no es así, sino más bien, lo que se afirma, es que la duración del mundo antes de la creación del sol, es igual a la duración de este número de revoluciones anuales del sol. La mente que una vez tuvo tanto tiempo como el anual revolución del sol, puede aplicar esa medida a la duración en la que no existía (Fraser, 1959).

Bridgeman (1965), hace una acotación del evento dentro de la concepción del tiempo y su uso, y escribe: El examen de uso muestra que el "evento" es un concepto de gran generalidad, aplicándose a muchos tipos diferentes de situación física. Sin embargo, en todos sus usos siempre tiene una connotación temporal e implica, un "suceso" de algún tipo. No es probable que se hable de un libro que descansa pasivamente sobre una mesa como un "evento (Bridgeman, 1965). Así que al menos, algunos eventos son estados de muy corta duración. Aun así, se debe considerar, que algunos eventos son cambios de estado: ¿tendremos que contar? ¿Estas cosas entre las entidades básicas relacionadas por las relaciones temporales? Pero un cambio de estado es simplemente el caso limitante de un proceso; es un paso a través de una serie de estados con solo dos miembros (Von Wright, 1963). En consideración a las anteriores conceptualizaciones, se denomina tiempo a una medida efectuada sobre los cambios que se producen en el universo. Así, desde tiempos antiguos la filosofía se ha abocado con diversas posturas al análisis de este tópico, y más cercana a la presente época, lo ha hecho con mucha probidad la física, en especial desde la teoría de la relatividad. 
El tiempo independiente de su abordaje teórico-histórico, siegue siendo una realidad existencial dentro de la sociedad actual, y aunque las anteriores conceptualizaciones reflejan cierta relatividad en cuanto a su concepción general, se podría argumentar, que el tiempo, hace parte de la percepción que tiene cada persona sobre la realidad y el instante en que vive cada situación particular. A partir de la anteriores consideraciones o posturas teóricas, el tiempo hace parte de los recurso vivenciales del hombre, es decir, cada actividad que realiza el ser humano, está enmarcada por un antes, un ahora y un después, y más allá de su definición, toma relevancia su aplicación, valoración o abuso en las actividades que se realizan en cualquier esfera de lo social o individual.

Algunos estudios han analizado un conjunto de datos en diferentes períodos de tiempo sobre la inversión de tiempo académico de los estudiantes universitarios en los Estados Unidos entre los años de 1961 y 2003 , y los hallazgos han determinado que los estudiantes emplean menos tiempo en el año de 2003 frente a la inversión en horas del año de 1961, se considera, que las disminuciones fueron extremadamente significativas, por tanto, han habido cambios sustanciales en el tiempo en la cantidad o forma de producción de capital humano en los campus universitarios (Babcock and Marks, 2011). Existen también estudios que contraponen los resultados anteriores, es decir, de que en la sociedad moderna los individuos poseen menos tiempo para desarrollar sus actividades diarias. Uno de esos estudios, fue desarrollado por Robinson and Godbey (2010), quienes exponen, que el factor tiempo actual puede ser de mayor abundancia, comparado con otras épocas en la sociedad estadounidense, en ese sentido, consideran que en promedio los norteamericanos actuales, presentan más de una hora de tiempo libre por día que en la década de los 60.

En el tema del aprovechamiento del tiempo por parte de los estudiantes, se puede concebir, que los retrasos de los horarios de clases, pueden impactar significativamente en el rendimiento académico, la salud y la seguridad de los estudiantes (Watson, et al., 2017). De allí, la pertinencia de que haya una conciencia pública sobre los peligros de los horarios de inicio temprano y los beneficios de los horarios de inicio posteriores, y en parte ello, está en línea con el manejo adecuado del tiempo por parte de los estudiantes universitarios. La mayoría de las escuelas públicas norteamericanas ofrecen 10 días para las vacaciones, ene se sentido se considera, que un largo descanso tiene un gran potencial beneficioso. De igual manera, María Montessori, exponía acerca de la importancia del tiempo de inactividad, en donde es esencial que los estudiantes tengan tiempo libre para procesar y practicar las lecciones que aprendieron durante los períodos académicos (Maldonado, 2018). El uso y el abuso del tiempo se han convertido en factores que encierran un problema vivencial del individuo de la sociedad actual. El factor uso del tiempo, hace referencia fundamentalmente, a que se aproveche de la mejor forma el tiempo libre, es decir, las horas que una persona posee para desarrollar una actividad (sea social o individual), se realicen con la racionalidad debida, en el cual, puede planificarse dicho tiempo para sacarle mejores resultados.

Por otra parte, en cuanto al abuso, las muchas ocupaciones de las personas derivadas de la dinámica del mundo actual, no permiten que se aproveche dicho recurso en actividades que benefician el desarrollo personal o de la sociedad en su conjunto, y un ejemplo de ello, es la adicción que ha surgido en el presente siglo, en donde un significativo número de individuos, se pasan muchas horas frente a un dispositivo electrónico, y desaprovechan dicho tiempo, en el aprendizaje, el conocimiento o en la compartición con amigos o familiares. Este abuso del tiempo, no se había concebido por los antiguos pensadores, e inclusive los modernos, debido que la tecnología no había llegado con fuerza en la sociedad humana. Siguiendo con el abuso del tiempo, este puede significar pérdida de tiempo de vida, en el sentido, de que las personas se ocupan en muchas tareas que no son esenciales para su vida, y son más bien arte del ocio o del consumismo que está latente a nivel global. Para el caso de los estudiantes en su etapa de formación universitaria, la problemática que gira en función del tiempo en cuanto a su uso, se remite al hecho de que no hay una planificación del mismo para el estudio, así como tampoco, existe una orientación para sacarle mayor provecho a tal recurso, debido a que puede ser un buen aliado en dicha formación académica. En relación a su abuso, sin dudas, las nuevas tecnologías de la información y la comunicación, han sido un factor que ocupa mucho el tiempo de los estudiantes, y ello hace, que se dedique menos tiempo para el desarrollo de otras labores de importancia en ellos.

El presente artículo, está referido a precisar sobre cuál es la razón fundamental por la que los estudiantes de arquitectura no utilizan bien el tiempo en sus actividades académicas, y dicha indagación implica, el identificar las causas o distractores que se convierten en impedimento para que se haga un manejo inteligente de dicho recurso en este nuevo milenio. El artículo es de tipo descriptivo-reflexivo, en el cual, se valora el uso del tiempo, y ello conlleva a que los estudiantes universitarios se concienticen, acerca de la necesidad de una planificación adecuada, y el conocimiento indicado sobre la conducción del tiempo y de cómo podrían mejorar su uso. En el desarrollo del artículo, se abordan temas como el valor del tiempo, su aspecto psicológico, el real, la preempción, planificación, gestión del tiempo y el manejo del estrés, para 
luego evidenciar, acerca de un trabajo de campo y cuyos resultados conllevan a la elaboración de las conclusiones finales del artículo.

\section{Valor del tiempo}

Analizar la calidad del valor del tiempo, orienta la importancia de organizarlo y administrarlo bien, haciendo énfasis en la planificación, la cual es considerada como una clave fundamental para su máximo aprovechamiento. Para sacarle mayor partido a situaciones que lo requieran, se hace esencial estructurar cada actividad dentro de un marco de territorio compartido, estando esté relacionado no solo con el proceso de organización de los eventos, sino también con la forma como se establecen aproximaciones entre las personas involucradas y los programas establecidos y así planificar en forma efectiva las acciones requeridas en cualquier campo del conocer, del saber y del hacer. Reflexionando sobre la planificación, Arsham (2012), explica, sobre las restricciones, las cuales son determinadas por el (tiempo definido), y las restricciones probabilísticas (que serían, el evitar excederse del tiempo previsto), dan como resultado un buen tiempo programado con posibilidades que produzcan utilidades tanto en su buen manejo como en el cumplimiento de la labor y de los objetivos trazados.

\section{Tiempo psicológico}

El segundo considerado es el tiempo psicológico, el cual está en relación con la vivencia interna de las personas. Berne (1985), expone, que después de la necesidad de ser estimulados y de ser reconocidos, las personas precisan de programación, hablan de tiempo, de tiempo de aburrimiento, aprovechar el tiempo y perder el tiempo, detener el tiempo, programar el tiempo. El eterno problema en el uso apropiado del tiempo, está relacionado con una sistematización no enfocada, donde el primer paso consiste en definir de manera clara los efectos que se esperan obtener. Se recomienda entonces, que la definición del objetivo planteado responda a los requerimientos de los involucrados especificando por qué se hace importante sistematizar y cuál sería la forma apropiada de disfrutar el buen uso de la información, su disposición y resultado.

\section{Tiempo Real}

Cada movimiento en el tiempo está regido por uno o varios sistemas, siendo el sistema en tiempo real uno de las formas de interactuar y ejecutar actividades y tareas en intervalos definidos. Los elementos del tiempo real buenos de destacar son: a) Aspectos de unificación y rendimiento, b) Administración de situaciones difíciles, c) Manejo de indagación actualizada y en tiempo vigente, d) Elaboraciones y enunciado en lapso real, y e) Coordinación y notificación de trabajos. A esto se le suman una serie de errores que podrían presentarse antes, durante y después de su ejecución, como son el exceso en el tiempo requerido para las entregas y respuestas, con penalización por el incumplimiento de plazos. Las entregas y respuestas después del tiempo requerido, ignorándose completamente su contenido.

También se mencionan algunos de los problemas que podrían presentarse con el uso del tiempo real en actividades de toda índole serían, dificultades y cambios de argumento, la notificación sin sincronización de trabajos, la gran cantidad de datos variados y difíciles de manejar, el mal manejo de los errores y recuperación de arbitrajes y el procesamiento de información sin concordancia. Por lo tanto se hace urgente estructurar dicha información a través de metodologías apropiadas que ayuden a que fluya y que sea debidamente organizada, ordenada y seleccionada para su utilización futura. Se activaría la tarea teniendo en cuenta lo siguiente: a) Manejo de las actividades, b) Trabajos con límites de plazos en su ejecución, c) Control en el intervalo de dicha ejecución, d) Tiempo de arranque y terminación de dicha tarea o actividades, e) Tiempo de respuestas.

Las acciones deben estar adecuadamente estudiadas para que ocurran dentro un plazo debidamente especificado. Se recomienda entonces la flexibilidad, considerando que en algunas ocasiones se pueden perder plazos y de igual forma prolongarse los tiempos en dichas interrupciones. Existen factores que afectan el comportamiento temporal de un sistema, estos comportamientos están relacionados, con la ejecución de una actividad muy particular que tiene valores máximos y valores mínimos, dependiendo estos en gran medida de los recursos con que se cuente y de los canales de comunicación que agilicen sus acciones para su completo desempeño.

\section{Fenómeno Preempción}

Hay que evitar programar tareas que sean interrumpidas, llamando al fenómeno preempción, significando esto cuando se deja una tarea o actividad, por otra más prioritaria. El cambio de tarea o cambio de contexto, introduce en la vida de las personas una sobrecarga de tiempo, afectando su tiempo de respuesta. Por lo tanto se hace necesario trabajar por conseguir un tiempo nulo entre una tarea y otra, dándoles tiempo a las 
personas para reorganizarse y volver a concentrarse en las tareas definidas con plazos de ejecución que generan ajustes a las actividades del momento. Canedo (2010), en su texto Derecho Comunitario (20092010), menciona que el principio de "preempción" resulta de aplicación en las materias en las que tanto las comunidades como las entidades reguladoras deben ser competentes, sin poner en peligro las acciones futuras de cada uno de los grupos involucrados. La cantidad de tiempo asignado para realizar y procesar una tarea o acción, debe ser igual a su tiempo máximo de ejecución. Planificar el tiempo es posible si logramos finalizar cada acción en su límite temporal, es decir, cumpliendo los términos de tiempo; entonces, un buen planificador puede elegir entre minimizar los tiempos de los trabajos y el número de tareas de manera eficiente, para así hacer que se cumplan tanto la programación como las etapas en cualquier proceso planteado.

\section{Clasificación de la Planificación}

Dentro de la clasificación de la planificación tenemos la planificación cíclica, que está relacionada con un alto grado de determinismo y la planificación estática, relacionada con procesos lentos y trabajos a largo plazo, requiriendo más tiempo para el desarrollo de sus actividades. Algunos de los problemas que se pueden presentar en la planificación del tiempo están relacionados con los siguientes ítems: a) La poca flexibilidad en la entrega de una actividad, cada vez que se modifica una tarea, se hace conveniente modificar el tiempo de su ejecución, b) Si las tareas o las acciones, son de distinta magnitud, se le deben asignar ciclos secundarios de ejecución, c). También se recomienda dividir una tarea en varios secciones y a través varios procedimientos, y de igual forma sincronizar las tareas con el tiempo disponible para su realización y d) Como elemento adicional para fortalecer las tareas y acciones se tiene el buen manejo de la información y la utilización de recursos que ayuden al buen aprovechamiento del tiempo. Revisando lo que expresa Ortiz (2004), existen tendencias muy marcadas en países subdesarrollados sobre la poca valoración del tiempo, considerando su mal manejo, la falta de centralidad en la tarea (estudio y trabajo), y la irresponsabilidad sobre las acciones concretas, como situaciones culturales que deberían ser pasadas por alto.

\section{Gestión del Tiempo}

El problema de la idiosincrasia en Colombia en lo relacionado con el mal manejo del tiempo, está ilustrado a través de Hoyos y Herrera (2008), cuando explican que la responsabilidad en el uso de la mayoría de los valores es intuito persona y no está relacionada con el que hacer de una comunidad y mucho menos un país o región. Estos autores, ilustran en su texto, que cualquier tipo de responsabilidad está considerada como un valor de vínculo y no como estereotipos. La delegación de logros en una persona es muy importante ya que vincula permanentemente a su estado de conciencia, a su definición de bien y mal, de correcto e incorrecto.

En la formación como humanos, no existe ningún escenario donde se enseñe a tomar conciencia de la importancia de cómo manejar adecuadamente el valor del espacio y del valor del tiempo. Cabe considerar que en estos dos campos son inevitables a los largo de la vida; siendo que no podría haber vida si no hay tiempo y espacio por el cual transitar. Desde la universidad Francisco de Victoria, Segura (2004), comparte 10 claves para aprovechar mejor el tiempo: a) Cada persona debe cuidar cada parte de su vida, gestionando bien el tiempo, y dedicándole tiempo a todas las áreas que se hacen necesarias considerando que (algunos requieren más dedicación que otras), b) No malgastar el tiempo, más vale 10 Min concentrado en una tarea que una hora sin estar atento(a) en ella, c) No postergar tareas innecesariamente, sin excusas, hay que hacer lo que se tenga que hacer en el momento que debe estar hecho, d) Alcanzar los objetivos que se propongan, ser eficientes, economizar los recursos y el tiempo, e) Evitar los ladrones del tiempo. cuáles son tus ladrones del tiempo?, entre los más comunes se encuentran: Las interrupciones, falta de organización, confusión en las prioridades, pesimismo en la valoración de las propias habilidades, potencial de trabajo, escasa delegación de tareas, postergación de tareas, precipitación en la toma de decisiones, entorno desordenado, falta de planificación, urgencias respuestas, dispersión, falta de disciplina, planificar y organizar el tiempo, decidir cuánto tiempo hay que dedicarle a cada día, como eres y no como te gustaría ser, revisar porque nunca se cumple lo que se planifica, ser proactivos, que sería adelantarse a lo que podría ocurrir (Universia.es, 2012).

\section{Manejo del estrés}

Es claro en los trabajos de Klipling (1894) y Dogson (1865), que el sujeto no puede interactuar en el escenario social sino se compromete con sus normas, lenguajes, símbolos y valores, sin embargo es la clase dominante la que establece parámetros que minimizan la efectividad de las personas, creándoles exceso de responsabilidades las que se ven traducidos a una de las enfermedades de mayor incidencia en el siglo XXI, como es el estrés. Uno de los factores que afecta el manejo del tiempo es estrés (Gallego, 2013), por tanto no se debe retrasar la ejecución de tareas. 


\section{METODOLOGIA}

La metodología es de tipo descriptiva, en la cual se evidencian los resultados de una encuesta dirigida a un grupo de universitarios en una universidad privada de la ciudad, y cuyo propósito, es el de identificar, las causas o distractores que se convierten en impedimento para que los estudiantes no hagan un manejo inteligente del tiempo, A continuación, se describen los resultados, que se derivan de la selección de una muestra al azar no probabilística para la realización de una encuesta a 31 estudiantes de V y VI semestre de arquitectura, entre edades 19 y 26 años.

\section{RESULTADOS}

La Tabla 1, muestra las situaciones descritas por los estudiantes de arquitectura, para explicar el uso y el abuso de su tiempo. En la tabla, no sólo se aprecian los elementos que le quitan tiempo a los estudiantes, sino que también, se visualiza una falta de conciencia de la razón del porque esas actividades influyen negativamente. Con base en la tabla ya descrita, se puede argumentar, que el sentido de justicia alrededor de la utilización del tiempo es mal interpretado, debido a que sólo se manifiesta, comparado con la carencia o demasía de elementos materiales. Otro punto a destacar, es porque si a los estudiantes le sobra demasiado el tiempo, porque no utilizan ese tiempo en superar sus debilidades. Cada persona que tiene que utilizar el tiempo para cualquier actividad que lo requiera, debe estar nivelada y asimilando todo lo relacionado con su deber moral y permanente, en su campo personal. Para el caso de los estudiantes de arquitectura, es notorio, el mal manejo del tiempo, y el desaprovechamiento del mismo, se debe considerar, que el uso o abuso del tiempo no solo se remite a la ocupación de tareas o el desarrollo de ciertas actividades sociales, sino también, es un recurso que permite la reflexión, concientización y la construcción humana, y en procura de mejorar el tiempo de vida, tanto de la propia persona como de su prójimo.

\section{DUSCUSION FINAL}

El concepto del tiempo es complejo y relativo tal como lo demuestra la discusión filosófica que gira alrededor de esta categoría. En un estado comparativo de tal concepto, para Thomas de Aquino, el tiempo se concibe, como aquel movimiento que ocurre en un antes y un después. En cambio, para Platón, el tiempo es un proceso. Por su parte Aristóteles sostiene, que no se puede percibir el tiempo como tal, por tanto, solo se es consciente de él, a través de su paso, es decir, solo a través del cambio o movimiento discernidor. En la concepción del tiempo, Newton, aporta que todas las cosas son colocadas en el tiempo en orden de sucesión; y en el espacio como por orden de situación, ello conlleva, a que se puede medir el tiempo. De igual forma, Barrow, sostiene que el espacio y el tiempo existen con independencia de los cuerpos materiales o acontecimientos físicos, pero no independientemente de Dios. De lo anterior, se puede concluir, que el entendimiento del tiempo, no logra definirse con precisión, sino más bien, en cuanto a la relación de este elemento establece con las demás cosas del universo.

Es bueno señalar que con la ayuda de una planificación sobre la conducción del tiempo y con un estado de conciencia elevada se podría mejorar su uso. El mal manejo del tiempo se da en las personas, cuando no encuentran el equilibrio necesario para organizar sus actividades. En el caso de los estudiantes universitarios de la facultad de arquitectura, en la mayoría de los casos, estos terminan no haciendo nada o dejando de hacer las tareas más importantes y significativas. Otra situación está relacionada con hacer actividades distintas a las planteadas, donde un distractor lleva a las personas a otra dimensión en la cual pierden la razón real de dicha tarea.

Según Steel (2011), en su libro Procrastinación, la predisposición se debe a la interacción entre el sistema límbico (comprometido de tramitar emociones como el placer, miedo, recompensa y excitación) y la corteza prefrontal (que se encarga de la organización y toma de medidas). Según el autor, la procrastinación se produce cuando el sistema límbico veta los planes a largo plazo de la corteza prefrontal y se inclina por hacer cosas más inmediatas. Steel considera que la procrastinación está en los genes. Para su supervivencia, los antepasados les venía muy bien tener una mente impulsiva. Esta condición denominada procrastinación, puede ser algo explicativo de porque los estudiantes universitarios no planifican el uso del tiempo de manera adecuada, debido a que se dejan seducir de la inmediatez de las cosas, como las nuevas tecnologías, y ello puede que no les permita un mayor afianzamiento de su mente en cuanto a valorar las cosas que haya que dedicarle más tiempo por ser más importantes para su vida.

Existen también situaciones que contribuyen a empeorar la relación de las personas con el tiempo, como tener demasiadas cosas que hacer, retrasar el término de una tarea, esperar a que les digan lo que tienen que hacer, no poder terminar porque no tienen todas las respuestas entre otras. Para dar respuesta a cuál sería el comportamiento apropiado para el uso del tiempo se hace necesario que cada persona lo comprenda, lo conozca y pueda enfrentarlo a su realidad, asumiendo los retos que implique cada actividad o tarea planteada, buscando las estrategias apropiadas para su aprovechamiento. Tomar conciencia de su 
realidad, indagar sobre metodologías adecuadas y mejorar el rendimiento, entender la mayoría de las dinámicas de trabajo y buscar la forma de optimizarlas.

Tabla 1: Metodología y muestra sobre el uso del tiempo en los estudiantes de Arquitectura. Fuente: Delma, 2014

\begin{tabular}{|c|c|c|c|}
\hline NNo & Género & Edad & Situaciones \\
\hline 1 & $\mathrm{H}$ & 24 & $\begin{array}{l}\text { Falta de concentración, desinterés, no querer realizar algo, desmotivación, falta de estímulos } \\
\text { y alicientes, problemas. }\end{array}$ \\
\hline 2 & $\mathrm{H}$ & 21 & $\begin{array}{l}\text { Ruido del exterior, uso del celular, impuntualidad, indisciplina, incomodidad en el área de } \\
\text { trabajo, ambientes inadecuados para el área de estudio. }\end{array}$ \\
\hline 3 & $\mathrm{H}$ & 21 & $\begin{array}{l}\text { Falta de concentración, sueño y desnutrición, falta de planificación y compromiso con su } \\
\text { proceso de aprendizaje, desmotivación, falta de estímulos y alicientes, distracciones, } \\
\text { elementos distractores (objetos tecnológicos, mal uso). }\end{array}$ \\
\hline 4 & $\mathrm{H}$ & 19 & $\begin{array}{l}\text { Falta de planificación, Falta de interés, agotamiento, acostarse tarde, dormir poco, pensar } \\
\text { lento, impuntualidad, distracción, elementos distractores, objetos tecnológicos mal uso. }\end{array}$ \\
\hline 5 & $\mathrm{H}$ & 20 & $\begin{array}{l}\text { Uso exagerado de las redes sociales, salir con los amigos sin planearse, ver televisión } \\
\text { cuando hay otras obligaciones que cumplir, dormir hasta tarde sin planificación, distracción, } \\
\text { distractores, objetos tecnológicos mal uso. }\end{array}$ \\
\hline 6 & $\mathrm{H}$ & 20 & $\begin{array}{l}\text { Practicar deportes de manera exagerada, rumba sin planificación, uso exagerado de las redes } \\
\text { sociales, dormir más de la cuenta, mal manejo del ocio con más tiempo del necesario }\end{array}$ \\
\hline 7 & $\mathrm{H}$ & 20 & $\begin{array}{l}\text { Vida social exagerada, uso exagerado de las redes sociales, ver televisión cuando hay otras } \\
\text { obligaciones que cumplir, dormir más de la cuenta, mal manejo de elementos distractores, } \\
\text { objetos tecnológicos mal uso. }\end{array}$ \\
\hline 8 & $\mathrm{H}$ & 22 & $\begin{array}{l}\text { Tiempo lento de respuesta, uso exagerado de las redes sociales, juegos en línea OP7, pasar } \\
\text { tiempo fuera de la casa, sin planificación ni objetivos definidos, distracción por medio de } \\
\text { objetos tecnológicos. }\end{array}$ \\
\hline 9 & M & 22 & $\begin{array}{l}\text { Falta de concentración, malos hábitos dormir hasta tarde, sentarse en la calle a ver pasar a la } \\
\text { gente, ponerse a jugar cartas o dominó, uso excesivo del teléfono. }\end{array}$ \\
\hline 10 & $\mathrm{H}$ & 19 & $\begin{array}{l}\text { Relaciones sentimentales, malas amistades, redes sociales, rumba sin control, actividades } \\
\text { innecesarias. }\end{array}$ \\
\hline 11 & $\mathrm{H}$ & 22 & $\begin{array}{l}\text { Redes sociales, vídeo juegos, amistades, relaciones sentimentales, rumba sin control, dormir } \\
\text { más tiempo del necesario. }\end{array}$ \\
\hline 12 & $\mathrm{H}$ & 21 & Móvil, perder el tiempo no haciendo nada, halterofilia, TV, quehaceres. \\
\hline 13 & $\mathrm{H}$ & 20 & TV, internet, fútbol, videojuegos, pereza. \\
\hline 14 & $\mathrm{H}$ & 20 & Familia, redes sociales, amigos, pereza. \\
\hline 15 & $\mathrm{H}$ & 21 & $\begin{array}{l}\text { Sin objetivos definidos, apatía a la carrera, videojuegos, pereza y estado de confort } \\
\text { prolongado, redes sociales. }\end{array}$ \\
\hline 16 & $\mathrm{H}$ & 20 & El no saber por dónde empezar, distractores, hambre, dormir más de la cuenta, pereza. \\
\hline 17 & $\mathrm{H}$ & 20 & Redes sociales videojuegos, rumba sin planificar, TV, pereza. \\
\hline 18 & $\mathrm{H}$ & 19 & Música, amigos, vídeos anime, películas, TV, parejas. \\
\hline 19 & $\mathrm{H}$ & 20 & Facebook, TV, pereza, rumba sin planificar, parejas. \\
\hline 20 & $\mathrm{H}$ & 22 & Fútbol, TV, internet, rumba sin planificar, videojuegos. \\
\hline 21 & $\mathrm{H}$ & 19 & La música, TV, vídeo juegos, dormir más de la cuenta, fútbol. \\
\hline 22 & $\mathrm{H}$ & 18 & Licor, música, ir a la calle sin necesidad, amigos ociosos, dormir más de la cuenta. \\
\hline 23 & $\mathrm{H}$ & 26 & $\begin{array}{l}\text { Malas compañías, no tener claro los objetivos o metas, sin rumbo, sin compromisos en la } \\
\text { vida, no pensar e el futuro solo en el ahora. }\end{array}$ \\
\hline 24 & $\mathrm{H}$ & 19 & Alcohol, malas amistades, pereza flojera, TV, redes sociales. \\
\hline 25 & $\mathrm{H}$ & 18 & Internet, falta de motivación, TV, relaciones sentimentales, alcohol. \\
\hline 26 & $\mathrm{H}$ & 20 & Internet, videojuegos, problemas familiares, rumba sin control, relaciones amorosas. \\
\hline 27 & M & 19 & $\begin{array}{l}\text { Hacer las cosas que no me divierten ni apasiona, sin metas ni sueños, ser pesimistas, dormir } \\
\text { más de la cuenta, aplazar las cosas. }\end{array}$ \\
\hline 28 & M & 19 & Pereza, TV, inseguridades, falta de motivación. \\
\hline 29 & M & 19 & Sin organización, cansancio, compromisos sin planear, redes sociales, celulares. \\
\hline 30 & M & 19 & Redes sociales, TV, celular, falta de organización, pereza. \\
\hline 31 & M & 18 & Facebook, depresión, falta de interés, sin planeación. \\
\hline
\end{tabular}




\section{CONCLUSIONES}

De acuerdo al trabajo presentado y a los resultados obtenidos, se pueden plantear las siguientes conclusiones principales:

1.- Existe una subvaloración del tiempo por parte de los los estudiantes universitarios de la facultad de arquitectura, y esta percepción sesgada de dicho recurso hace que estas personas no dediquen el tiempo suficiente para su formación integral.

2.- La falta de planificación en el uso del tiempo por parte de los estudiantes universitarios no facilita el que se aproveche al máximo dicho factor, y no se pueda logra mayor eficiencia del mismo, debido a que este recurso se ha convertido en algo limitado debido a las múltiples ocupaciones en que vive el ser humano en la actualidad.

3.- En relación al abuso del tiempo, los resultados obtenidos en la investigación, coinciden con la afirmación general, de que las nuevas tecnologías de la información y la comunicación son los mayores distractores, que hacen que los estudiantes desaprovechen este recurso para su ocupación hacia el conocimiento y de la compartición con familiares y amigos.

4.- Es pertinente, que los estudiantes universitarios desarrollen estrategias, métodos y recursos para hacer una aplicación inteligente del tiempo, y ello conlleva al final, a que haya una mayor valoración en cuanto a su uso y evitar en lo posible su abuso.

\section{REFERENCIAS}

Arsham, H., Perturbation Analysis for IS Project Management Based on a Single Time Estimate, International Journal of Enterprise Information Systems, 8(4), 77-98 (2012).

Robert, G.A., The Leibniz-Clarke Correspondence. Manchester, Eng.: Manchester University Press (1977).

Babcock, P., y Marks, M., The Falling Time Cost of College: Evidence from Half a Century of Time Use Data. Review of Economics and Statistics, Vol 93. Issue 2, p.468-478. Harvard College and the Massachusetts Institute of Technology (2011).

Blackwell, et al. Aquinas, T., Commentary on Aristotle's "Physics., trs. New Haven: Yale University Press, Bk. IV, 17, sec. 577. 7, Physics (1963).

Bridgeman, P., Sophisticate's Primer of Relativity. New York: Harper \& Row, p. 115 (1965).

Berne, E., La intuición y el Análisis Transaccional. Sevilla, Jeder Libros (2010).

Burtt, E., A The Metaphysical Foundations of Modern Science. New York: Anchor Books, Chap. V. (1932).

Cajori, F., Sir Isaac Newton's Mathematical Principles of Natural Philosophy and His System of the World. Berkeley: University of California Press, pp. 6, 8 (1960).

Canedo, J., Derecho Comunitario (2009-2010). Revista de Derecho Comunitario Europeo No 12 (2010).

Dogson, C., Alicia en el País de las Maravillas (1865).

Gallego, C., Domina tu tiempo 2. Incrementa tu productividad. Haz más. Gana más dinero. 2013. Disponible en la web: https://www.freelibros.me/videotutoriales/domina-tu-tiempo-carlos-gallego-video-y-audio-curso

Van, F.C., An Introduction to the Philosophy of Time And Space. Nousoul Digital Publishers (2013).

Child, J. M., The Geometrical Lectures of Isaac Barrow tr. La Salle, III.: Open Court, pp. $35-37$ (1916).

Hope, R., Physics, Lincoln: University of Nebraska Press, 217b, 30-244a, 20 (1961).

Kipling, R., El Libro de la Selva (1894). Editorial: Macmillan Publishers. Inglaterra (1984).

Locke, J., An Essay Concerning Human Understanding, A. C. Fraser, ed. New York: Dover, Bk. II, xiv, 24 (1959).

Maldonado, N. Importance of Free Time for Students. Arthur Morgan School's (2018). Available on the web https://www.arthurmorganschool.org/importance-of-free-time-for-students/.

Robinson, J, y Godbey, G., Time For Life: The Surprising Ways Americans Use Their Time (2010)

Steel, P., Procrastinación. Porque dejamos para mañana lo que podemos hacer hoy. ISBN: 9788425343964 (2011).

Von Wright, G. H., Norm and Action. London: Routledge \& Kegan Paul ; New York : Humanitie (1963).

Watson, N., y otros 12 autores, Delaying middle school and high school start times promotes student health and performance: an American Academy of Sleep Medicine position statement. J Clin Sleep Med. 2017;13(4):623-625.

Zubiri, X., El Concepto Descriptivo del Tiempo. Realitas II: 1974-1975, Trabajos del Seminario de Cael. 283, b26, pp. 747, Madrid (1976). 